\title{
Performance Optimization by Task Scheduling in Cloud Computing
}

\author{
Amit Kaushal, Sarvpal Singh
}

\begin{abstract}
Cloud computing is associate on demand access to a shared pool of resources. Vendor's of cloud computing offer application and change technology, infrastructure, hardware, software, and integration for consumer. The most aspect of cloud computing is accessibility and performance of their network association. It refers to the method of allocating users' tasks to virtual machines (VMs) with a goal of minimizing the work time and rising the resource utilization. Tasks programming is taken into account NP onerous drawback with $O(m, n)$ run time complexness to schedule $n$ tasks on $m$ resources. A computer hardware adapts its programming strategy consistent with the ever-changing setting and therefore the variety of task. We provide comparison with Max-min scheduling formula and Genetic formula. Our hybrid algorithm provides higher performance compared to different programming algorithm.
\end{abstract}

Index Terms: Task scheduling, Cloud computing, Auction based task scheduling algorithm, Genetic algorithm, Max-min scheduling Algorithm.

\section{INTRODUCTION}

Cloud computing is the transportation of computing services like servers, storage, databases, networking, software, analytics and more over the web based on pay as you use model. Cloud computing is a computing method based on the Internet, by this way, the sharing of hardware resources and information can be provided according to requirements to computers and other equipment Cloud computing still underneath in its development stage and has several challenges. Out of the different issues in cloud, scheduling plays exceptionally vital part in deciding the compelling execution .There has been varied forms of scheduling algorithm existing in cloud system and task scheduling is one of them. Scheduling oversees accessibility of CPU memory and great planning arrangement gives greatest utilization of asset by mapping of task with particular VMs [1, 2,]. Associate economical task programming mechanism is often needed not solely to keep up the turnout, however conjointly helps the service supplier to keep up the conditions of SLA's by providing the great QoS that optimizes the performance of cloud computing [23]. The Cloud Computing Model is given below in figure 1 .

Revised Manuscript Received on October 18, 2019.

Amit Kaushal, Department of Computer Science \& Engineering, MMMUT, Gorakhpur, India.

Sarvpal Singh, Professor \& Head, Department of ITCA, MMMUT, Gorakhpur, India.

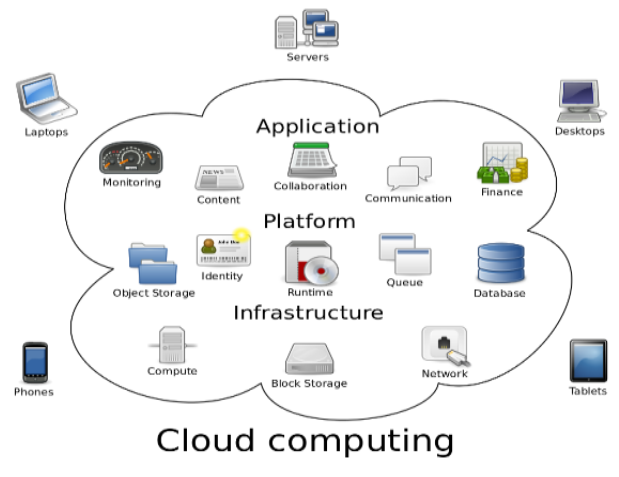

Figure 1:Cloud computing model.

Tasks scheduling steps are modeled as illustrated in Figure.2.

1. A user of CC submits a task to a scheduler.

2. A scheduler communicates with Cloud Information System (CIS) for obtaining data concerning resources.

3. CIS provided the resources data to the scheduler.

4. The role of scheduling algorithm is to mapping task to the proper resource and submits the task to the winner resource.

5. The user gets the identification (id) of the resource and uses it through cloud interface.

6. The user sends the input file to the resource consistent with the schedule.

7. The scheduler gets over time updated data concerning the standing of a cloud to manage the schedule.

8. The knowledge is distributed to the user.

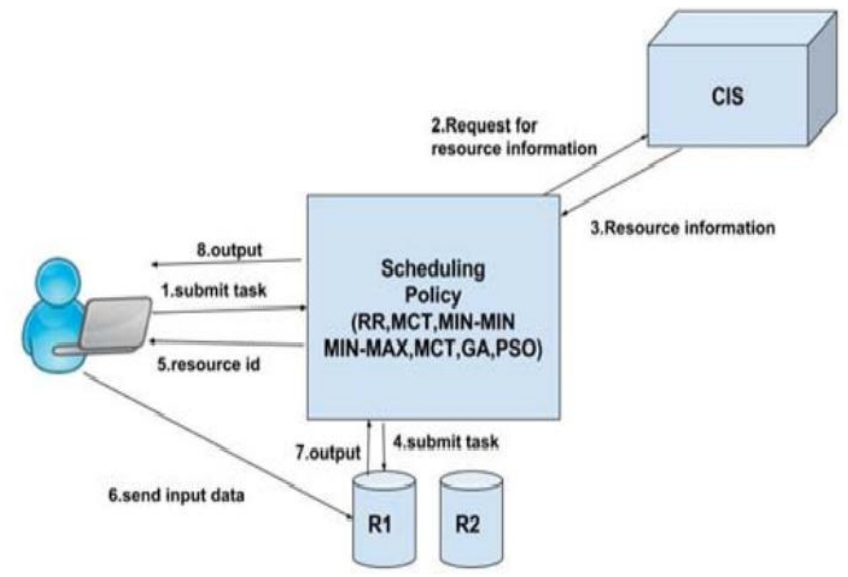

Figure 2: Process of Task Scheduling.

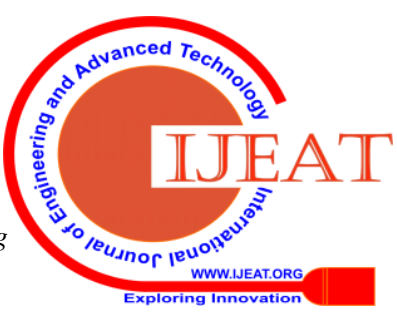




\section{Performance Optimization by Task Scheduling in Cloud Computing}

\section{LITERATURE REVIEW}

- In 2010 Van den Bossche et al. projected associate optimum tasks programming policy in hybrid cloud model [2]. The hybrid consists of each non-public and public cloud. The situation of the matter is that some workloads must be outsourced from private cloud to public cloud throughout the height load intervals wherever there aren't any enough resources privately cloud that cowl the submitted user's tasks. These workloads are affected by a point and QoS needs. During this case, a call creating method is required to pick out that workloads to source and to what cloud supplier, in such some way the use within the internal information center is maximized and therefore the price of running the outsourced tasks is reduced.

- In 2011, Sindhu, S. and Saswati Mukherjee projected two algorithms for programming tasks in cloud computing [3] supported the process needs of a task and therefore the machine potential of a resource. The primary formula, named Longest Cloudlet fastest process component (LCFPE), tries to make less the makespan by distribution of lengthier cloudlets to a process components (PEs) having high machine power. The second formula, named Shortest Cloudlet fastest process component (SCFP), will the other.

- Gogulan, R., A. Kavitha, and U. Karthick Kumar, in 2012, given a brand new nature impressed formula [5] referred to as multiple Pheromone Algorithm (MPA) that belongs to ACO algorithms. MPA generates dynamic schedule that the task is completed in minimum time and therefore the resource utilization is increased. MPA achieved higher QoS than ACO and algorithms consistent with 3 studied parameters: makespan, price and reliableness constraints.

- Ravichandran, S. and E. R. Naganathan, in 2013. They applied genetic formula to unravel the matter of uncertainty in tasks arrival to the cloud [4]. This drawback leads to tedious binding for tasks to VMs. The projected plan to unravel this drawback was dynamic programming wherever arrived user's tasks are queued and therefore the computer hardware role is to kind them supported the computation and memory usage; then, GA is employed to choose every task and realize the simplest fit allocating a task to offered virtual machines and acquire the global improvement.

- In 2014, Agarwal, Dr. and Saloni Jain given in their work, for task programming in $\mathrm{CC}$, new formula, named generalized priority formula (GPA) [7]. The formula was experimented and compared with FCFS and RR algorithms for variable range of VMs and employment traces and victimization CloudSim machine. The results show that the projected formula was a lot of economical than FCFS and RR algorithms.

- In 2015, A. Branch, K. conducted a research using Cuckoo improvement primarily based Task programming formula [9]. During this formula, bird nets simulate the process units (virtual machines) and eggs are the tasks. The cuckoo's role is to get eggs (tasks) within the nets (VMs). Victimization this methodology and supported the amount of VMs and therefore the tasks number (inputs of the algorithm) varied orders of those machines are examined every time till tasks are allotted to hosts within the right manner.
- In 2016, Hamad, Safwat A., and Fatma A. Omara given associate improved genetic formula (TS-GA) for task programming drawback within the cloud computing setting [10]. The aim of the projected formula was to reduce the completion time, and maximize resource usage and results was simulated victimization CloudSim machine and therefore the results showed reduced price, improvement in resource utilization, increased speedup, and higher ratio for algorithm efficiency when put next with default GA and RR algorithms.

- In 2017, Mondi, Syed Hamid Hussain compared the performance of six rule primarily based heuristic algorithms for tasks programming supported parameters [12] like price, makespan and turnout victimization CloudSim. These algorithms are FCFS, MCT, MET, MaxMin, MinMin and Suffer age. The MinMin formula performed higher than different heuristics.

- In 2018, Bappaditya Jana and Jayanta poray developed a hybrid application [21] by combining PSO and genetic. Thought-about to enhance the performance in multi cloud having fragmented interfaces, evaluation schemes and virtual machine offerings.

\section{PROPOSED FRAME WORK AND METHODOLOGY}

A. Genetic Algorithm: In 1975, Holland introduced the employment of Genetic formula [10] for associate optimality method for the primary time. Later in 1989, this approach was completed by Goldberg. The invention of this formula as associate improvement algorithm had been impressed by the speculation of evolution. Genetic algorithms are far-famed to for his or her stability and potency for locating the world optimum resolution in an exceedingly very complicated and huge sample area [15]. They're also helpful find the optimum resolution for each single objective furthermore as multi-objective issues. So we tend to like it in task programming in cloud.

Basically Genetic algorithm includes six steps as:

- Initialization

- Use of fitness function

- Selection

- Crossover

- Mutation

- Accept and Replace.

The quality genetic formula (SGA) can be exploited to unravel the matter of task programming in cloud. The steps for this are given below:

1. Initialize population- The produced population is indiscriminately victimization binary cryptography wherev er every body corresponds to a VM and therefore the genes of this chromosome represents the regular tasks on this VM. Example on this VM1[ T4,T2,T5],VM2[T1,T7,T6],VM3[T3,T8,T9]

2. The fitness function- The aim associated with programming tasks on VMs is to search out the simplest assignment of tasks on $\mathrm{VMj}$ specified the completion time for tasks on VMs is reduced. This could be developed

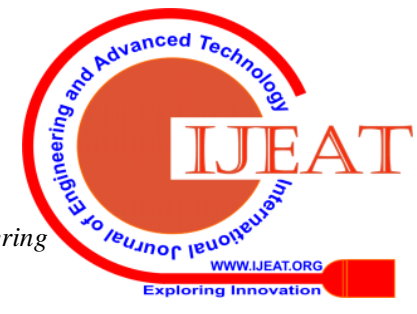


victimization the subsequent equation.

$\mathrm{Pj}=\sum \mathrm{Pij}$ where

$\mathrm{Pj}$ is the execution time of all tasks $(1 \ldots \mathrm{n})$ on $\mathrm{VMj}$.

$\mathrm{Pij}$ is the execution time of task Pi on VMj.

$\mathrm{Pij}=\mathrm{Ci} / \mathrm{Psj}$

Where $\mathrm{Ci}$ is the computation complexity of tasks $\mathrm{Pi}$ and Psj is the processing speed of $\mathrm{VMj}$.

3. Selection process- During this step the 2 random people are chosen to try and do the GA operations on them so as to come up with new population and therefore the nonelected individuals are unbroken untouched.

4. Crossover- It's the method of exploring the search area and generating new solutions (descendents) kind the initial solutions (parents) by interchanging the genes of the chosen chromosomes.

5. Mutation- It's to try and do operations like swap, move or replacement on sequence values.

6. Evaluation- The solutions are evaluated supported the fitness functions and therefore the ones that achieved smart fitness are chosen within the next iteration.

B. Auction Based Task Scheduling Algorithm: This algorithm was initial projected by Dimitri Bertsekas in 1979. The term "auction formula" applies to many variations of a combinatorial improvement algorithm that solves assignment issues [24], and network improvement issues with linear and convex/nonlinear price. Associate auction formula has been employed in a business setting to work out the simplest costs on a group of merchandise offered to multiple patrons. It's associate unvarying procedure, wherever multiple bids are compared to work out the simplest supply, with the ultimate sales visiting the best bidders.

The auction based Task Scheduling mechanism involves following steps:

Step1. Customers submit requests and bids.

Step2. If submission finishes, still Step three. Otherwise repeat Steps one and a pair of.

Step3. Sort bids.

Step4. Verify the winner consistent with the auction and mapping the resources to the winners.

Step5. If the auction's stopping condition is happy, still Step six. Otherwise, repeat step five. The stopping condition, reckoning on the auction model used. As an example, all VMs are allotted and every one requests have been happy, etc.

Step6. The winner pays for the VMs; the winning value conjointly depends on the auction model used.

Step7. Bind the VMs to the winning Task (i.e., the winner).

\section{PROPOSED NEW HYBRID APPROACH FOR TASK SCHEDULING}

Genetic algorithm has exceptional effects on global searching ability however usually causes low efficiency and huge quantities of redundancy iteration. Auction formula converges to optimum resolution quickly in later stage. This paper combines blessings of 2 algorithms, and puts forward task scheduling algorithm referred to as Genetic-Auction primarily based hybrid algorithm. The proposed model of the GABHA algorithm

\section{PROPOSED MODEL}

During this proposed Model of Task scheduling, the tasks are appointed resources consistent with genetic formula supported separate result that concerning which resource is to be appointed to which task. Genetic formula relies on the biological construct of populace era. The task that happy fitness operate of genetic formula can goes underneath the biding method of Auction primarily based programming Mechanism. During a double-sided auction each cloud customers and cloud suppliers will submit bids and asks, severally. The trade are often dead now once a customer's bids and a provider's asks match or are compatible with associate auction primarily based task programming framework for cloud computing, the purchasers put forward requests for resources with bids and therefore the winner are allotted the resources by employing a stated auction mechanism. So the task are mapped with resources like processor, memory, storage etc...Through Virtualization Techniques. During this approach Performance is optimized in higher than model through hybrid task scheduling algorithm. The Proposed Model of the GABHA algorithm shown above in figure 3 .

Genetic algorithm has exceptional effects on global searching ability however usually causes low efficiency and huge quantities of redundancy iteration. Auction formula converges to optimum resolution quickly in later stage. This paper combines blessings of 2 algorithms, and puts forward task scheduling algorithm referred to as Genetic-Auction primarily based hybrid algorithm. The flowchart of the GABHA algorithm above in figure 4. The Genetic-Auction primarily based Hybrid formula (GABHA) is especially divided into 2 sections: within the initial phase, initializes the population indiscriminately and applies GA operator's tournament selection (TOS), replica (crossover), and mutation consecutive. The second section applies the Auction methodology over GA to induce optimum solutions. The GABHA formula is associate improvement algorithm that consolidates the options of GA and Auction algorithms to boost the performance of Task scheduling.

\section{PROPOSED ALGORITHM}

//phase 1: Genetic Algorithm

Step 1: Initialize population randomly

Step 2: GA method

//Apply GA operators

2.1: Tournament selection (TOS)

(Depending on relative fitness)

2.2: Reproduction

(Apply PC, carry out crossover)

2.3: Mutation

(Using PM)

Step 3: Repeat until the termination criterion reached //phase 2: AUCTION Algorithm

Step 4: Auction Method

// apply bidding process of auction on Task 


\section{Performance Optimization by Task Scheduling in Cloud Computing}

\section{1: Sort bids}

4.2: Match VM to potential winners

4.3: The winner task get the VM

// If termination condition satisfied

Step 5: Repeat until the termination criterion reached and Get best solution.

\section{IMPLEMENTATION AND EXPERIMENTAL RESULTS}

In this paper, we've used cloudsim for simulation [25, 26]. Our projected formula is intended in cloudsim layer. Every benefit ask from cloud client is named as assignment which must be recorded by Virtual machines. The task Simulation range diverse from 100 to 600 with unvarying augmentation by 100 . Consistent with Table one, we can understand that our given strategy provides relatively less execute time than max-min and Genetic formula. The proposed-scheduling formula provides minimum scheduling length by $1.009 \%$ than max-min programming and $1.059 \%$ than Genetic formula for 300 numbers of tasks which is shown below in Fig. 5. Equally in Table two, we can also see that our projected strategy provides relatively less execute time than max-min and Genetic. The Proposed hybrid-scheduling algorithm provides maximum successful execution ratio by $1.039 \%$ of max-min programming and $1.081 \%$ than genetic algorithm for 200 task that is shown below in Fig.6.

\begin{tabular}{|c|c|c|c|c|}
\hline \multicolumn{2}{|c|}{ TABLE I. } & \multicolumn{3}{|c|}{ MEAN SCHEDULING LENGTH } \\
\hline $\begin{array}{l}\text { Number } \\
\text { of } \\
\text { iteration }\end{array}$ & $\begin{array}{l}\text { Number } \\
\text { of task }\end{array}$ & $\begin{array}{l}\text { Max-Min } \\
\text { algorithm } \\
\text { time }\end{array}$ & $\begin{array}{l}\text { Genetic } \\
\text { algorithm }\end{array}$ & $\begin{array}{l}\text { New hybrid } \\
\text { task } \\
\text { Scheduling } \\
\text { algorithm }\end{array}$ \\
\hline 1 & 100 & 360 & 320 & 270 \\
\hline 2 & 200 & 750 & 698 & 620 \\
\hline 3 & 300 & 1120 & 1070 & 1010 \\
\hline 4 & 400 & 1472 & 1410 & 1350 \\
\hline 5 & 500 & 1746 & 1715 & 1660 \\
\hline 6 & 600 & 2064 & 2010 & 1930 \\
\hline
\end{tabular}

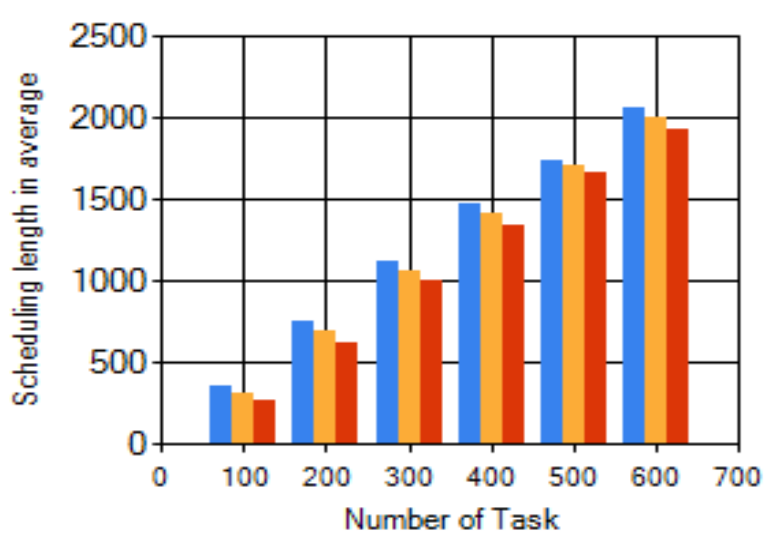

Max-Min Scheduling

Genetic Algorithm

New Hybrid Algorithm

Figure 5: Mean scheduling length.
TABLE II. SUCCESSFUL EXECUTION RATIO

\begin{tabular}{|r|r|l|l|l|}
\hline $\begin{array}{l}\text { Number } \\
\text { of } \\
\text { iteratio }\end{array}$ & $\begin{array}{l}\text { Number } \\
\text { of task }\end{array}$ & $\begin{array}{l}\text { Max-Min } \\
\text { algorith } \\
\text { m time } \\
\text { algorith } \\
\text { m }\end{array}$ & $\begin{array}{l}\text { Genetic } \\
\text { hybrid task } \\
\text { Schedulin } \\
\text { g } \\
\text { algorithm }\end{array}$ \\
\hline 1 & 100 & 0.53 & 0.52 & 0.55 \\
\hline 2 & 200 & 0.51 & 0.49 & 0.53 \\
\hline 3 & 300 & 0.48 & 0.48 & 0.51 \\
\hline 4 & 400 & 0.47 & 0.48 & 0.49 \\
\hline 5 & 500 & 0.44 & 0.45 & 0.49 \\
\hline 6 & 600 & 0.4 & 0.42 & 0.48 \\
\hline
\end{tabular}

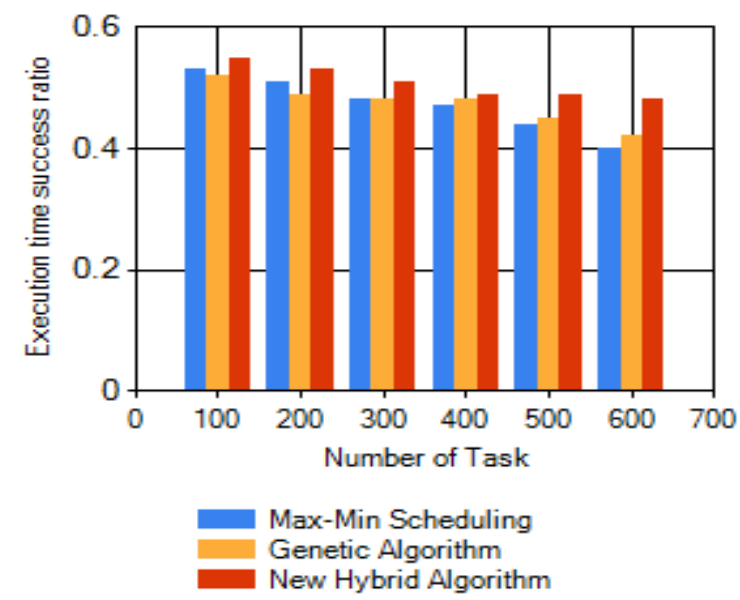

Figure 6: Successful execution ratio

\section{CONCLUSION}

Our proposed algorithm provide stronger latent period from cloud supplier and reduce the holding up time for a specific shopper in cloud. Cloud computing could be a distributed primarily based computer paradigm that is employed by users to induce smart quality with less price. As task scheduling could be a challenge in cloud computing, totally different algorithms are prompt and applied to induce higher results concerning utilization of system resources, latent period and satisfaction of user demands. During this study, we tend to highlight a number of the task programming algorithms employed in cloud supported by examples, specifically genetic formula and Auction primarily based task scheduling algorithm. We tend to studied the behavior of recent Hybrid task scheduling inside the setting of CloudSim and compared between the performance of the Max-min scheduling formula and Genetic algorithm according to average holding up time. The results show, once victimization new Hybrid algorithm to scheduling range of Cloudlets over number of VMs, the average waiting time becomes less than Max-min, using the identical numbers of cloudlets and therefore the $\mathrm{CC}$ environments. For future work, it's price to research the impact of different parameters like VMs, datacenters, memory, information measure for network and storage in cloud environments, and replicate that in real physical environment.

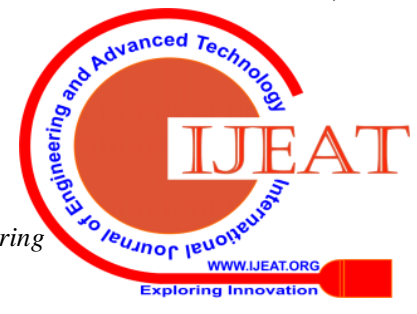




\section{REFERENCES}

1. Niloofar Khanghahi and Reza Ravanmehr "Cloud computing performance evaluation: Issues and challenges" International Journal on Cloud Computing: Services and Architecture (IJCCSA).Cloud Computing: Services and Architecture (IJCCSA), Vol.3, No.5, October 2013 DOI 10.5121/ijccsa.2013.3503.

2. Van den Bossche, R., Vanmechelen, K., \& Broeckhove, J. (2010, July). Cost-optimal scheduling in hybrid Iaas clouds for deadline constrained workloads. In Cloud Computing (CLOUD), 2010 IEEE 3rd International Conference on (pp. 228-235). IEEE.

3. Sindhu, S., \& Mukherjee, S. (2011). Efficient task scheduling algorithms for cloud computing environment. In High Performance Architecture and Grid Computing (pp. 79-83). Springer, Berlin, Heidelberg.

4. Ravichandran, S. and E. R. Naganathan, "Dynamic Scheduling of Data Using Genetic Algorithm in Cloud Computing", Integrated Intelligent Research (IIR), International Journal of Computing Algorithm Vol 02, Issue 01, June 2013, Page No.11-15 ISSN: 2278-2397.

5. Gogulan, R., Kavitha, A., \& Kumar, U. K. (2012). "A multiple pheromone algorithm for cloud scheduling with various QOS requirements". Int. J. Comput. Sci, 9, 3.

6. Kaleeswaran, A., Ramasamy, V., \& Vivekanandan, P. (2013). "Dynamic scheduling of data using genetic algorithm in cloud computing". Park College of Engineering and Technology, Coimbatore, India.

7. Agarwal, D., \& Jain, S. (2014). Efficient optimal algorithm of task scheduling in cloud computing environment. arXiv preprint arXiv:1404.2076.

8. Lakra, A. V., \& Yadav, D. K. (2015). Multiobjective tasks scheduling algorithm for cloud computing throughput optimization. Procedia Computer Science, 48, 107-113.

9. Branch, K. (2015). A novel task scheduling method in cloud environment using cuckoo optimization algorithm. International Journal of Cloud-Computing and Super-Computing, 2(2), 7-20.

10. Hamad, S. A., \& Omara, F. A. (2016). Genetic based task scheduling algorithm in cloud computing environment. International Journal of Advanced computer Science and Applications, 7(4), 550-556.

11. Dandhwani, Vanita, and Vipul Vekariya."Evolutionary Algorithm Using K-mean For Task Scheduling in Cloud Computing."

12. Madni, S. H. H., Latiff, M. S. A., Abdullahi, M., \& Usman, M. J. (2017). Performance comparison of heuristic algorithms for task scheduling in IaaS cloud computing environment. PloS one, 12(5), e0176321.

13. Alia Teresa T M, Niyas Ibrahim and K R Ramesh Babu "Latency aware scheduling policy for tasks in IaaS cloud" 2013 International Conference on Recent Trends in Information Technology (ICRTIT) on 25-27 July 2013 pp.725-731 ISBN:978-1-4799-1024-3/13/\$31.00 @2013 IEEE.

14. Tani, Hicham Gibet, and Chaker El Amrani. "Smarter round robin scheduling algorithm for cloud computing and big data." Journal of Data Mining and Digital Humanities (2018)

15. Jang, S. H., Kim, T. Y., Kim, J. K., \& Lee, J. S. (2012). The study of genetic algorithm based task scheduling for cloud computing. International Journal of Control and Automation, 5(4), 157-162.

16. Ashutosh Ingole, Sumit Chavan, Utkarsh Pawde. "An optimized algorithm for task scheduling based on activity based costing in cloud computing" (NCICT) 2011, Proceedings published in International Journal of Computer Applications® (IJCA).

17. Zhong, H., Tao, K. and Zhang, X. 2010 "An Approach to Optimize Resource Scheduling Algorithm for Open-Source Cloud Systems" The Fifth Annual China Grid Conference.IEEE Computer Society, 978-0-7695-4106-8.

18. Tawfeek, M., El-Sisi, A., Keshk, A. and Torkey, F.2015. Cloud Task Scheduling Based on Ant Colony Optimization. International Arab Journal of Information Technology, Vol. 12, No. 2, 2015.

19. Gomathi, B. and Karthikeyan, K. 2013. Task scheduling algorithm based on hybrid Particle swarm optimization in Cloud Computing environment. Journal of Theoretical and Applied Information Technology, September 2013. Vol. $55 \mathrm{~N}^{\circ} 1$.

20. Goyal, T., Singh, A., \& Agrawal, A. (2012). Cloudsim: simulator for cloud computing infrastructure and modeling. Procedia Engineering, 38, 3566-3572.

21. Bappaditya Jana and Jayanta poray " A Hybrid Task Scheduling Approach Based on Genetic Algorithm and Particle Swarm Optimization Technique in Cloud Environment" Advances in Intelligent systems and computing 695, Springer Nature Singapore Pte Ltd. 2018, https://doi.org/10.1007/978-981-10-7566-7 61.

22. Mohit Agarwal and Dr. Gur Mauj saran Srivastava."A Genetic Algorithm inspired task scheduling in Cloud Computing" International Conference on Computing, Communication and Automation (ICCCA 2016 IEEE), ISBN: 978-1-5090-1666-2/16.
23. Ruba Abu Khurma, et.al "Task Scheduling Algorithm In Cloud Computing Based On Modified Round Robin Algorithm" Journal of Theoretical and Applied Information Technology $15^{\text {th }}$ sep.2018.Vol.96. No 17. ISSN: 1992-8645.

24. Michael M. Zavlanos, Leonid Spesivtsev and George J. Pappas,"A Distributed Auction Algorithm for the Assignment Problem" Proceedings of the 47th IEEE Conference on Decision and Control Cancun, Mexico, Dec. 9-11, IEEE 2008, 978-1-4244-3124-3/08.

25. Buyya, R., Ranjan, R., Calheiros, N.: Modeling and Simulation of scalable cloud computing environments and the cloudsim toolkit: challenges and opportunities. In: Proceedings of the 7th High Performance Computing and Simulation Conference, Leipzig, Germany, pp. 1-11 (2009).

26. Mao, Y., Chen, X., Li, X.: Max-Min task scheduling algorithm for load balancing in cloud computing. J. Springer (2014).

27. K. Vijayakumar and C. Arun, "Continuous Security Assessment of Applications in Cloud Environment", International Journal of Control Theory and Applications, ISSN: 0974-5645 volume No. 9(36), Sep 2016, Page No. 533-541.

28. R.Joseph Manoj, M.D.Anto Praveena, K.Vijayakumar, "An ACO-ANN based feature selection algorithm for big data", Cluster Computing The Journal of Networks, Software Tools and Applications, ISSN: 1386-7857 (Print), 1573-7543 (Online) DOI: 10.1007/s10586-018-2550-z, 2018.

29. K. Vijayakumar and V. Govindaraj, "An Efficient Communication Technique for Extrication and Cloning of packets on cloud", International Journal of Applied Engineering Research, ISSN 0973-4562 Vol. 10 No.66 May 2015

\section{AUTHORS PROFILE}

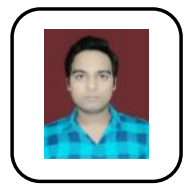

Name :Amit Kaushal.

Designation: Student.

Field of Specialization: Computer Network, Mobile \&

Cloud Computing, Database.

Email Id :amitkaushalmmmut@gmail.com

Highest Qualification : M.Tech

Publications : 2

Membership : IEEE Student member

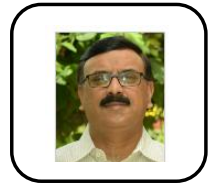

Name : Sarvpal Singh.

Designation : Professor \& Head.

Field of Specialization :Wired/Wireless networking, Mobile \& Cloud Computing, Linux OS

Email Id : singh_sarvpal@yahoo.co.in

Highest Qualification : Ph.D.

Publications/Books/Patents: 48

Membership \& Association of Academic IETE, CSI. 